\section{THE FORM OF COMETS*}

III.

WE are now arriving at a conclusion, for the conditions mentioned are very narrow. In order that a force exerted by the sun may present at any point whatever of the trajectory of a moving body these two radial and tangential components, it is sufficient, and it seems to me absolutely necessary, that this force should not be propagated instantaneously like attraction, but successively, i.e. at a definite rate. In order that it may drive to a distance the rarest materials of comets, at the same time exerting only an extremely, weak action upon their much more dense and more compact nuclei, it is necessary, and it is sufficient, that this force should be one of surface and not of mass, like attraction. If the light of the sun were idue, as was long believed, to the emission of innumerable atoms moving at the rate of 77,000 leagues a second, the force exerted by these atoms would fully satisfy these conditions. Unfortunately, the emission hy. pothesis has been shown to be false, and is now replaced by that of the undulations of an imponderable fluid on which attraction has no hold. If statical electricity, in order to produce attractions and repulsions, had no need of a particular material medium, such as our atmosphere, we might perhaps be able to call in the aid of that force : but it would still be necessary to prove that the sun is electrical, and that it is able to develop a very marked electric state in comets without acting similarly upon

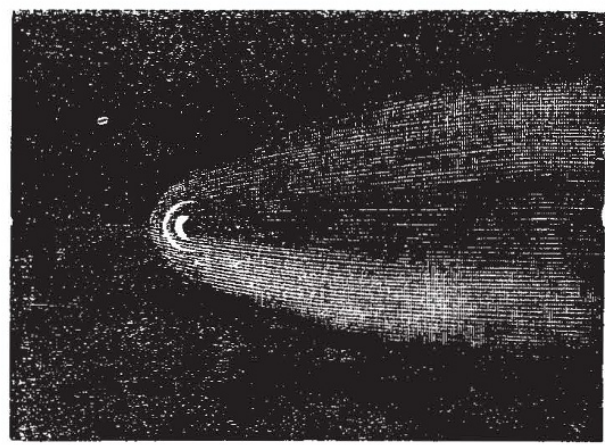

[Frg. 8.

other bodies. As to magnetism, which appears independent of any medium, we know very well that it is not a surface-action, but an entirely specific force, capable of attracting or repelling the densest materials; and besides, the phenomena of terrestrial magnetism scarcely leave room for attributing to the sun a magnetic power sensible at such distances. Finally, electricity and magnetism are polar forces which impart to bodies opposite powers of attraction and repulsion, while cometary phenomena argue only a simple repulsive force.

There remains the repulsive force of solar heat. In all bodies heat gives rise to a force among the molecules which tends to separate them more and more ; it is this which enables our steam-engines to work, and which forces projectiles from our guns. It is evidently a surface-action, and not one of mass ; and at least, in maintaining that it is sensible only between the molecules of bodies, i.e. that its sphere of action is infinitely small, it is natural to think that the surface of a heated body exerts its repelling action all round it, as well as towards the interior. Moreover, there is nothing opposed to the supposition that this force is propagated successively, since its cause, heat, is itselt propagated in planetary space with a definite speed, that of light.

Here is our hypothesis formulated. By introducing this repulsive force, acting by successive propagation, into

\footnotetext{
* Continued from p. $\$ 248$
}

the differential equations of the movement of comets, along with that of attraction, we see that there springs from them the established phenomenon of their acceleration with its most delicate characteristics.* The analysis which I made has been latterly revised and verified by an illustrious Italian geometer, M. Plana, with developments which leave nothing to be desired in point of mathematical rigour, while the hypothesis of a resisting medium starts, as I have said, with conditions incompatible with the principles of mechanics. It only now remains for us to see whether the same force will also account for the highly complicated phenomena of the figure of comets.

Let us set out from this distinctive characteristic :The repulsive force exercised at a distance by the incandescent surface of the sun is a surface-action, the more capable of driving off a body, the smaller the density of the body is. According to an estimate deduced from the observed acceleration of Encke's conet, it will be sufficient to reduce, in the proportion of $\mathrm{I}, 000,000$ to $\mathrm{I}$, the density of the nucleus of that comet to represent the excess of this repulsive force over attraction. The ques-

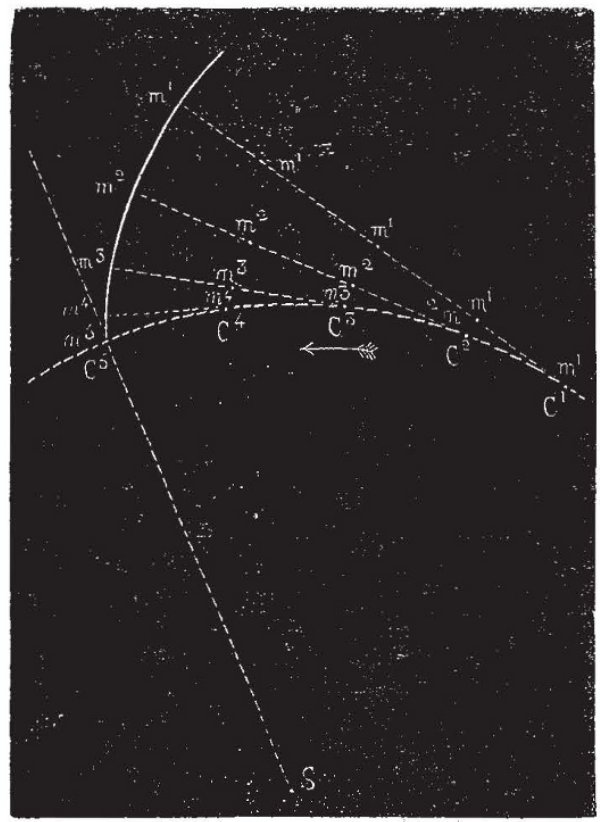

FIG. 9.

tion, then, is, to discover if such great variations of density are exhibited by the various appendages of comets, of which the most compact parts have already so small a mass under so enormous a volume. But this is precisely what facts establish in the most exact and striking manner. The figure of Donati's comet, which I am about to bring before you, shows that the nucleus, in proportion as it is subjected to the heating action of the sun, emits vapours which go on dilating more and more, so as to form around the nucleus envelopes having a radius ten or even a hundred times greater. But if the matter of a sphere with a radius equal to $I$ expands into a sphere having a radius equal to roo, it is sufficient to make the density become one million times less. In fact, all the matter of the nucleus is not thus disseminated in the head of the comet; this dilatation affects only a very small portion of the primitive mass, and we see how the density of the extreme layers of the head may fall much below the figure given by the above calculation.

* The progressive change of two elements alone, to wit, mean motion and eccentricity ; nothing on the position of the plane of the orbit; for the rest, simple periodic inequalities scarcely sensible, but differing, however, from
those which the resistance of a medium-ould give. 
WWe shall understand better what precedes by examining for a little in detail some phenomena presented by the head of the comet of 1858 , at the time when the already formed tail was continually fed by materials emitted by the nucleus, and carried away by solar repulsion. (See Fig. 8.)

The concentric zones of a decreasing brightness, which are noticed around the nucleus, on the side next to the sun, are due to an intermittent emission of matter. This matter is seen to dilate more and more with a very moderate initial speed of about is metres per second, and finally to reach the limits of the head of the comet; a second, a third, \&c. emission closely follow the first, and are developed in the same manner. The brightness, at first very marked, of these successive envelopes of the nucleus grows rapidly weaker in proportion as their density diminishes. Finally, in the exterior layers, the more and more rarefied materials become the prey of the solar repulsion, which makes them turn back, driving them towards the tail at a rate incomparably greater than the former, for in twenty-five days the tail of Donati's comet

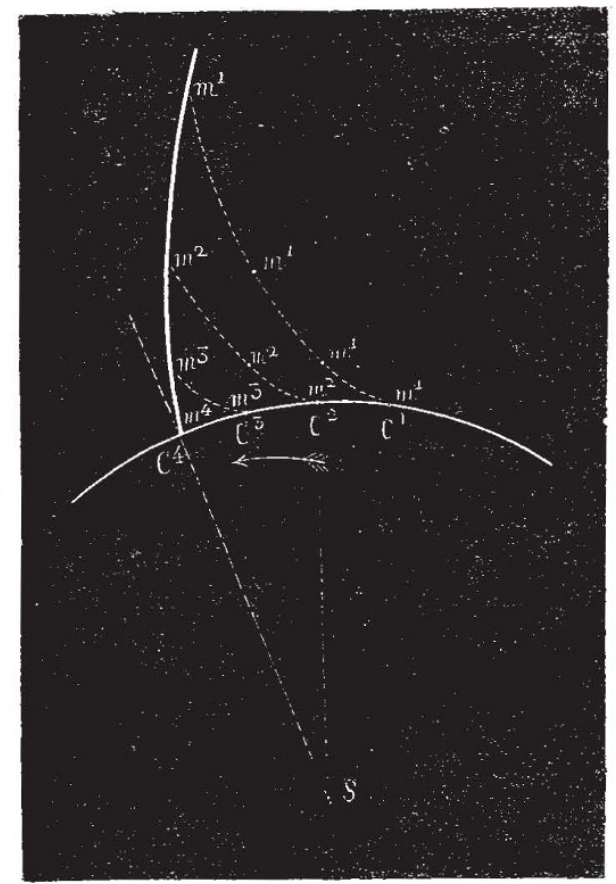

FIG. Io.

had reached a length of $14,000,000$ leagues; it increased in length at the rate, not of 19 metres, but of 8 leagues per second. I showed, at the outset, to what excessive rarefaction the materials of these immense appendages a'tain.

You see that upon such materials a surface-action like the repulsive force must have beautiful play, while the solar attraction, independent of the surface and density, continues to act in the same manner upon all these molecules. The struggle, then, between these two forces will turn in favour of the former as soon as the progressive dilatation of the cometary matter, gradually spreading itself in surrounding space, will have brought it to a certain degree of diffusion, and there is nothing to hinder the repulsive action thus becoming twice, three times, even ten times more powerful than attraction.

From the fact that this force, or rather that the radial component of this force, acts in the direction of the radius vector, from the fact that the expelled molecules preserve very nearly the tangential speed which the comet possessed, it necessarily results, as we shall see, that the tails, from the first, must be opposite to the sun and bent in a backward direction.

Fig. 9 represents the successive positions of a series of molecules emitted by the nucleus of a comet so as to constitute the axis of the tail. In this figure, we suppose for the molecules a density such that the repulsive force exactly counterbalances the solar attraction: thus their motion, solely due to the tangential velocity of the comet, takes place in a straight line. To simplify matters, this

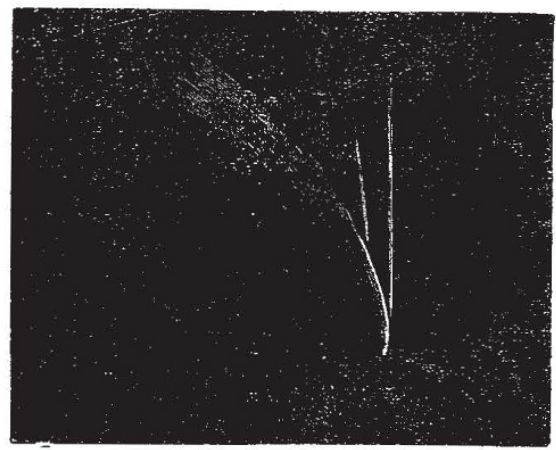

Fig, Ir.

rate has even been supposed constant, a; if the orbit were a circle.

On the first day, the comet being at $\mathrm{C}^{\mathrm{y}}$, a molecule $\mathrm{m}^{\mathrm{2}}$ is detached and subsequently follows the line $m^{1} m^{1} m^{1} \ldots$. On the second day, a molecule $m^{2}$, likewise leaves the nucleus at $\mathrm{C}^{2}$, and subsequently describes the tangent $m^{2} m^{2} m^{2}$....Similarly, on the third day, for a molecule $m^{3}$, and so on. If we join by a continuous line the series of positions occupied at the same time, the fifth day, by all these molecules $m^{5}, m^{4}, m^{3}, m^{2}, m^{1}$, we shall

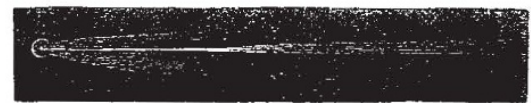

FIG.12

have the curvilinear axis of the tail ; this will be, in this particular case, the involuie of a circle. This constructicn accounts for the three laws which have been ascertained as the result of observation :- $\mathrm{I}$. The tail, at its origin, is sensibly opposed to the sun, $S ; * 2$. The tail is curved backwards on its path; 3. The axis of the tail is a plane curve situated in the plane of the orbit.

If the density of these molecules were still smaller, the repulsive force would prevail over the solar attraction, and these molecules would describe no longer straight lines.

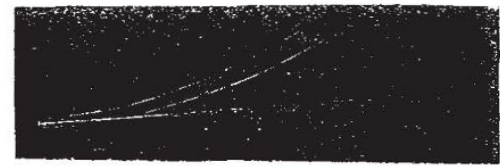

F1G, I3.

but sections of an hyperbola whose convexity would be turned towards their common focus, S. (See Fig. Io.)

The series of points $m^{1}, m^{2}, m^{3}, m^{4}$, emitted at $C^{1}, C^{2}$, $\mathrm{C}^{3}, \mathrm{C}^{4}$, by the comet, gives yet another curve like the former, but with a curvature much less pronounced and nearer to the radius vector.

There results from this theory a consequence to which

* In reality the axis of the tail is not rigorously tangential at $\mathrm{C}^{5}$ to the radius vector; it makes with this radius a small angle for which the theory accounts, but which I think may be neglected here for the sake of brevity and simplicity. 
I must call your earnest attention, for it is verified in nature in the most striking way and upon the largest scale. All molecules of the same density must naturally group themselves together in the vicinity of the curvilinear axis of the tail $m^{5} m^{4} m^{3}$. . . . and thus form the open plume to which we have referred; but if the coniet emit molecules of very unequal densities, on which the repulsive force acts with different energies, there ought to be several distinct tails, more or less curved, all situated behind the radius vector. This is precisely the case with Donati's comet. Fig. II proves the truth of this; it shows the comet with three distinct tails. The two smaller tails were almost straight, but always in rear of the radius vector; they presented their less marked convexity in the same direction as the bright tail.

The great comet of $186 \mathrm{I}$ had also two tails. When we saw it for the first time, on June 30 , it appeared to have only one, $118^{\circ}$ long and perfectly straight, except a singular irregularity for which we could not at first account (see Fig. I2). But soon the two tails separated, and it became evident that we had been deceived by a simple play of perspective. The earth, in fact, on June 30 was in the plane of the orbit of this comet, and as the curvilinear axes of the tails are always situated in this plane, they were united, from our point of view, into one and the same straight line, or at least into one and the same arc of the great circle of the celestial vault. The sketch of the same comet (Fig. 13) seen a fortnight previously by observers in the sow thern hemisphere, shows clearly the disposition of this double tail, the most curved half of which almost touched the earth with its extremity.

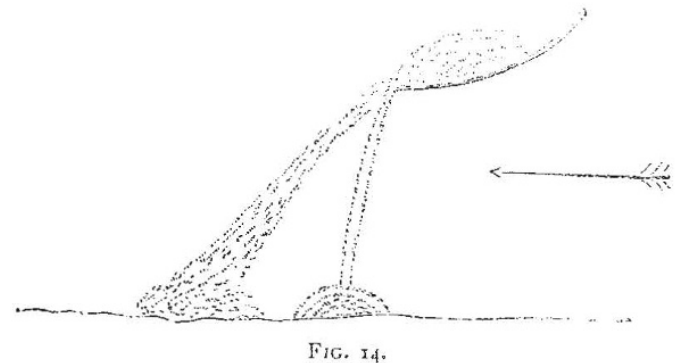

Fig. If.

These singular effects of the repulsive force are easily explained by a comparison which will appear at first to be far removed from our subject, but the fundamental analogy of which is palpable: I refer to the winnowing of corn. In fact, we cannot better compare the entire surface-action of the repulsive force than to that of a puff of air which repels light bodies and has no sensible action upon denser bodies. When we wish to separate the grain from the chaff by means of the winnowing fan, we allow both to fall gradually into a current of air; the grain escapes from its action and falls at the feet of the winnower, while the chaff, much lighter, is carried to a distance, and forms upon the ground a separate heap (see Fig. I4). If a third material, still lighter than the chaff, is found mixed with the grains placed upon the fan, it will be drawn away still farther, and will form a third heap beyond the second. Evidently the fall into space, under the sole influence of terrestrial attraction, would not operate with such discrimination, for all matters placed upon the fan would fall at the same rate and along the same curve, whatever might be their density.

Well, the repulsive force of the sun-a surface-action, and not one of mass, like attraction-winnows, so to speak, the materials which are separated from the cometary nucleus by being rarefied; it picks them out and distributes them, according to their density, into tails of different curvatures. The lightest form the straightest tails, and those nearest to the prolonged radius vector, while the nucleus, escaping the repulsive action on account of its relatively enormous density, continues to obey, almost rigorously, the Keplerian laws of attraction.

We need not believe that the phenomenon of muliple tails is rare; without speaking of the horrible dragon depicted in Fig. 2, many comets have had several tails. The facility with which the almost straight but very feebly luminous tails of Donati's comet escaped observers in France, leads us to believe that the phenomenon may be general, and that by careful inspection several tails may almost always be found to each comet. And according to theory, a perfect homogeneity of materials, the necessary condition for a single tail, must be, for any celestial body, rather the exception than the rule.

But then, it may be said, if very dense matters are drawn away by nucleal emission on the side next the sun, ought these materials escaping the repulsive action not to take the lead of the nucleus and form a sort of tail on the sicle next to the sun? Yes, without doubt; and this case is effectually fulfilled, for some rare comets have presented it, such as those of 1823,1845 , and $185 \mathrm{I}$. I would not insist upon these exceptional but not abnormal tails, situated on the side nearest to the sun, almost lying upon the orbit, or at least forming an obtuse angle with the initial direction of the ordinary tails.

\section{(To be continued.)}

\section{DR. BHAU DAFEE}

$T$ HIS very remarkable native of India, the true friend of his fellow-countrymen as well as of science and learning, died on May $3 \mathrm{I}$ at the comparatively early age of $5 \mathrm{x}$ years. As many of our readers may be ignorant of the claims of Dr. Bhau Dajee to notice, we give a brief sketch of his career, for which we are indebted to the Times of India.

He was born in 1823 in the village of Manjeren, near Sawunt Warree. His parents were in poor circumstances, and when he was about seven years of age they came to Bombay, bringing him with them. He was first placed in the native Education Society's Schools in Bombay, and aftervards went to the Elphinstone College. There he took a foremost place amongst the scholars, and was noted for his ability and unremitting application to his studies. The highest scholarships were taken by him, and he was specially rewarcled witb a gold medal. When his studies were concluded he was appointed assistant professor of chemistry and natural philosophy at the college. About this time ( 1842 ) a prize of 600 rupees was offered by Government for the best essay in English and Guzerathi on Female Infanticide. This prize Bhau Dajee gained, and the essay, which has since been published, has always been looked upon as one of the best contributions on that subject. He commenced his studies at the Grant Meciical College, under Dr. Morehead, in 1845 . The college had only then been established for a short time. His success here was again most marked, and gained for him the lasting friendship of many distinguished members of the medical profession. He received his diploma in $185 \mathrm{I}$. He soon created a name for himself as a clever and rising medical practitioner, and quickly found himself in possession of an extensive practice amongst all classes. His time was divided between his medical duties and his historical and philological researches. From the first he took a great interest in all public questions, especially those which affected the interests of his fellow-countrymen. He, with Dr. Birdwood, was instrumental in the establishment of the Gardens and Victoria and Albert Nuseum, Bombay. The Bombay Association too may be said to owe its existence to his energy; he was the first secretary, and always took a deep interest in the discussions of the society on Indian affairs and measures. A considerable portion of his income was expended in procuring rare and valuable MSS. from Cashmere, Orissa, Benares, and Southern India 